Meta

Journal des traducteurs

Translators' Journal

\title{
Former des traducteurs : pour qui ? pour quoi ?
}

\section{Jean-René Ladmiral et Marie Mériaud}

Volume 50, numéro 1, mars 2005

Enseignement de la traduction dans le monde

Teaching Translation Throughout the World

URI : https://id.erudit.org/iderudit/010654ar

DOI : https://doi.org/10.7202/010654ar

Aller au sommaire du numéro

Éditeur(s)

Les Presses de l'Université de Montréal

ISSN

0026-0452 (imprimé)

1492-1421 (numérique)

Découvrir la revue

Citer cet article

Ladmiral, J.-R. \& Mériaud, M. (2005). Former des traducteurs : pour qui ? pour quoi ? Meta, 50(1), 28-35. https://doi.org/10.7202/010654ar

\section{Résumé de l'article}

Le concept de traduction est polymorphe, tant en matière de formation que de marché. Cet article essaiera de lever les ambiguïtés du concept, pour clarifier l'offre de formation et sensibiliser le marché à la véritable traduction professionnelle. d'utilisation que vous pouvez consulter en ligne.

https://apropos.erudit.org/fr/usagers/politique-dutilisation/ 


\title{
Former des traducteurs: pour qui ? pour quoi?
}

\author{
JEAN-RENÉ LADMIRAL \\ Université de Paris-X-Nanterre, Paris, France
}

MARIE MÉRIAUD

ISIT, Paris, France

direction@isit.icp.fr

\begin{abstract}
RÉSUMÉ
Le concept de traduction est polymorphe, tant en matière de formation que de marché. Cet article essaiera de lever les ambiguïtés du concept, pour clarifier l'offre de formation et sensibiliser le marché à la véritable traduction professionnelle.
\end{abstract}

\begin{abstract}
The translation concept is polymorphous, whether it relates to training or to market. This paper endeavours to remove the ambiguities associated with this concept in order to clarify the curricula and make the customer aware of what professional translation really means.
\end{abstract}

\section{MOTS-CLÉS/KEYWORDS}

formation, concept de traduction, marché, traducteur professionnel, outils

S’interroger sur la formation des traducteurs revient à désambiguïser ce qui est en jeu dans l'acte même de traduire. Cela semblerait simple si, dans le même temps et plus fondamentalement, le concept de traduction lui-même ne faisait pas problème. C'est un concept polysémique, au point qu'il pourrait sembler flou à certains. En fait, il recouvre plusieurs réalités différentes: en sorte qu'il y a lieu d'y voir ce que nous appellerions volontiers un concept-valise.

D'abord, il y a tout ce qui n'est «pas encore» de la traduction: pures et simples équations lexicales du type pain = bread, éléments de transfert linguistique, procédures de sémantisation, figure de rhétorique, etc. (Ladmiral 1995). Il y aurait aussi tout ce qui n'est « déjà plus » de la traduction, comme la machine à traduire, c'est-à-dire la traduction automatique (TA) supposée livrée "clé en main», achevée (Fully Automatic High Quality Translation); mais, à vrai dire, le problème se pose maintenant de façon plus nuancée, plus complexe (cf. inf.). Et puis surgissent tous les problèmes passionnants que pose la traduction littéraire. Plus généralement, il y a l'échéance de ce qu'Antoine Berman a appelé avec à-propos la «traduction des œuvres », y subsumant non seulement la traduction littéraire, mais encore la traduction philosophique et la traduction des textes sacrés.

Sans minimiser bien sûr l'intérêt de cette problématique essentielle de la traduction, notre propos ici se limite à la dite «traduction technique» c'est-à-dire ce que l'on appelle: la «traduction professionnelle» ou la «traduction spécialisée» (voire la «traduction à orientation spécialisée», comme semblent préférer dire d'aucuns). On notera qu'ici aussi, y compris dans les milieux directement concernés, il y a une hésita- 
tion quant à l'étiquetage de ce dont il s'agit. Comme nous venons de le voir, l'opposition traditionnelle entre «traduction littéraire» et «traduction technique » est imprécise. Surtout, elle ne dit rien de la traduction elle-même ni de l'interférence qui se joue toujours plus ou moins entre les deux - ce à quoi font écho les enseignements de traduction générale dans nos cursus de formation. Au reste, pourquoi voudraiton que la traduction littéraire manquât à être elle-même professionnelle?

La polysémie du concept de traduction semble être à l'origine de malentendus qui ne sont pas purement intellectuels. On pourrait presque dire que la compréhension de la nature de la traduction est inversement proportionnelle à la connaissance que l'on a de la réalité de l'acte traduisant: moins on traduit, plus le fait de traduire paraît simple! Cela n'est pas sans incidences sur le plan professionnel, tant en ce qui concerne la demande de traduction que la mise au point du cahier des charges implicite à la commande. Paradoxalement, celui qui a le plus besoin de la traduction est conduit à se figurer qu'il n'en a pas besoin vraiment.

Inutile d'insister sur la bonne conscience des unilingues qui, ignorant ce qui est en jeu, considèrent que l'équivalence abstraite entre deux termes de langues différentes suffit à faire une traduction (voir sup.). Sans parler de la méconnaissance du simple fait que «la traduction n'est pas l'original» (Mounin, 1955). Plus généralement, ce qui est en cause, c'est l'«illusion de transparence traductive» (Ladmiral, 2002). Mais, là encore, cela ne reste pas sans conséquences sur le plan professionnel : il convient d'insister sur l'aller et retour permanent qu'une formation à la traduction doit mettre en œuvre entre, d'une part, les contenus d'une formation qui doit tenir compte des besoins du marché et, d'autre part, une formation du marché qui ne comprend pas toujours quel est son besoin. Le traducteur doit non seulement vendre sa traduction, mais sans doute lui faut-il aussi «vendre» ce qu'est une traduction et ce qui définit le besoin de traduction; en sorte qu'il lui faut être aussi un pédagogue, qui contribue à l'éducation du client et, plus largement, du marché.

En somme, du côté des formateurs sérieux et professionnels, la question de l'objectif de la formation apparaît simple: il s'agit de former les meilleurs traducteurs possibles, c'est-à-dire non seulement excellents dans leurs langues de travail, mais aussi formés dans un ensemble de compétences dont une traduction digne de ce nom exige la mise en œuvre. Il y faut bien sûr une formation à la terminologie, mais il ne suffit pas de savoir se servir des termes: encore faut-il ne pas tout ignorer du domaine auquel ils réferent (subject matter), ce qui implique à terme une véritable spécialisation «technique». Du coup, les langues de travail sont aussi nécessairement des langues de spécialités (language for special purpose). Mais l'essentiel, c'est de rendre compte pleinement du sens, de le «faire passer », ce qui suppose d'assumer consciemment et résolument le décalage qui existe nécessairement entre le texte original (To) et ses possibles traductions ( $\left.\mathrm{Tt}, \mathrm{Tt} \mathrm{t}^{\prime}, \mathrm{Tt} \mathrm{t}^{\prime} . ..\right)$. Or, ce sont les modalités de ce décalage qui font la qualité même de la traduction. À quoi s'efforcent de répondre les enseignements de traductologie que nous avons introduits dans nos cursus de formation: non pas bien sûr dans une perspective de recherche, mais dans une formation appliquée à la pratique traduisante. Du côté du client: si différents niveaux d'approfondissement de la traduction (traduction déchiffrée, traduction banalisée, traduction absolue... [Gouadec 2002: 337]) sont envisageables en fonction de ses attentes, il n'en demeure pas moins que la qualité de chacun de ces niveaux doit être irréprochable. Ne semblerait-il pas que tout le monde dût en être d'accord? 
Quels sont donc les problèmes posés à la formation des traducteurs par le marché?

Pour cela, nous ne chercherons pas à mettre en place une analyse sectorielle du marché ni à adopter une perspective de marketing: un grand nombre d'études sont faites et disponibles sur ces sujets. Notre approche est à clarifier l'expression des besoins en traduction, pour autant qu'un client qui ne sait pas qu'il a besoin de vous, ou qui ne sait pas qu'il doit faire appel à vous, est un client potentiel inaccessible à ce jour. C'est vers lui qu'il nous faut porter toute notre attention. Cela est d'autant plus vrai qu'il semble que dans le même temps l'explosion des besoins en traduction s'accompagne d'une diminution, au moins relative, des appels à des compétences de haut niveau.

Et là, il y a lieu de distinguer le point de vue du client néophyte et celui du client mal informé, voire désinformé.

L'extension des relations internationales, des échanges économiques et des relations culturelles a augmenté de façon considérable les besoins de traduction, ce qui va de pair avec une diversification de leurs champs d'application, que ce soit dans des domaines qu'on pourra dire nouveaux (comme tout le domaine informatique, par exemple) ou dans des domaines où ces besoins n'avaient pas été ressentis jusqu'alors (comme la documentation des PME, par exemple). Par là même, les clients potentiels ou réels n'étaient pas obligatoirement préparés à définir ces besoins et donc à faire intervenir des professionnels dans la résolution des problèmes qu'ils posaient.

Un certain nombre de confusions sont nées de cette situation, tant il est vrai que face à un problème nouveau le premier réflexe est de le ramener à un problème ancien et de lui appliquer la solution qui a marché. Ramener l'inconnu au connu est conforme à la logique cartésienne, et surtout c'est économique en temps et en énergie. À titre d'exemple, on pourra proposer le schéma d'analyse et de raisonnement qui suit:

a) problème nouveau: je dois impérativement communiquer vers l'Allemagne toute ma documentation;

b) je dois donc en assurer la traduction;

c) l'opération me rappelle les exercices de thème réalisés dans mon apprentissage de l'allemand au secondaire, et les difficultés que je rencontrais venaient de mon niveau médiocre en allemand.

d) Début de solution: pour résoudre mon problème, je dois trouver un bon "germaniste».

e) Résolution du problème: il y a dans l'entreprise un cadre, ou une secrétaire, ou un stagiaire... qui «parle allemand» (!); je vais lui demander de faire la «traduction».

Ce modèle de raisonnement peut se développer par cercles concentriques en fonction de la «sophistication» des réponses possibles: après le bilingue expérimenté «sur le tas ", vient le recrutement systématique du native speaker; et à terme, la formation de tout le personnel, l'appel aux traducteurs "pas cher», l'utilisation de logiciels, etc.

On pourrait donc conclure qu'en toute bonne conscience, la montée en puissance des besoins de traduction s'est accompagnée d'une diminution de la visibilité de l'expertise que met en ouvre l'acte même de traduire. En allant plus loin dans l'analyse, on peut supposer que cette invisibilité de l'expertise a toujours existé. On a souvent l'impression que le travail de traduction est ressenti comme une gêne, un frein. Il est à peine exagéré de dire que le travail du traducteur est assimilé à un pur et simple exercice de dactylographie! Le passage d'une langue à l'autre étant supposé automatique, quasiment réflexe. Ce n'est évidemment pas le cas, bien sûr, des milieux 
informés et des clients fortement impliqués et professionnels, mais cela reste une naïveté très largement répandue, là même où on ne s'y attendrait vraiment pas. Paradoxalement, on pourrait même dire que les choses semblent empirer, du fait même que l'on a très généralement pris conscience de l'importance des langues étrangères: une connaissance superficielle en langue étrangère amène certains à s'imaginer que ceux qui disposent d'une maîtrise plus importante sont du même coup à même de traduire d'emblée; c'est l'idée que traduire n'est pas un vrai travail. En effet, des comportements actuels de commande ou mieux encore de non-commande de traduction on peut, semble-t-il, déduire que l'expertise du traducteur, supposée reconnue par le passé, reposait en fait sur la reconnaissance d'une maîtrise de la langue étrangère qui était très valorisée et semblait inaccessible: c'est le phantasme du polyglotte. Cette maîtrise des langues étrangères étant aujourd'hui plus répandue et/ou plus accessible, l'expertise du traducteur s'est diluée dans toutes les formes de la maîtrise linguistique. Ce qui revient à dire qu'elle n'a jamais été réellement reconnue pour ce qu'elle était vraiment ou, plus exactement, qu'elle reposait sur un malentendu.

Il n'y a pas que la méconnaissance du néophyte, il y a aussi toutes les approximations et les hésitations propres au client mal informé, dont le jugement peut se trouver faussé par des habitudes mentales qui «font écran». Une autre ambiguïté de la traduction, en effet, vient de son enracinement historique dans la traduction littéraire, dans la «traduction des œuvres ». C'est, plus fondamentalement, dire que, pour beaucoup, la traduction est conceptuellement enracinée dans la référence à l'écrit et au texte. Les grandes problématiques de la traduction sont nées avec l'écrit; et on est fondé sans doute à penser que ce sont les mêmes problèmes qui se posent à toutes les opérations de transfert de sens. Or, dès que les transferts de sens qu'opère la traduction intègrent la dimension de l'image, de l'audiovisuel, du multimédia, d'une part, et de l'informatique, d'autre part, on tend à ne plus parler de "traduction», mais d' «adaptation », de «localisation», etc.

Les exemples sont multiples où le traducteur professionnel se trouve confiné dans un rapport exclusif à l'écrit, au risque de mettre en péril l'excellence qualitative $\mathrm{du}$ "produit» terminal. Telle entreprise de localisation, par exemple, donne les textes écrits aux traducteurs et confie les «boutons» pour être «localisés» par un informaticien! Autre exemple: le responsable du bureau de la traduction du gouvernement canadien disant que le terme de traduction était invendable, au point que le fait d'être un bureau de la traduction interdisait de vendre des compétences en localisation. Ou encore: ce sont des entreprises de publicité qui donnent du texte au traducteur pour le traduire, pendant que eux, «adaptent»l'image - avec toutes les erreurs que cette division du travail dysfonctionnelle peut engendrer.

Mais il n'est nullement assuré que les données fondamentales du problème en soient pour autant brutalement bouleversées. Changer de concept n'est pas le signe d'un changement de problème.

Ce qui vient d'être dit sur la suprématie de l'écrit n'exclut pas bien sûr que, parallèlement et depuis longtemps, des instituts de formation comme l'ISIT et ceux de niveau analogue que fédère la CIUTI intègrent la formation à la «traduction orale», c'est-à-dire à l'interprétation, dans leur cursus. Cela reste perçu comme une spécialisation terminale et sélective, de très haut niveau, et qui est pour ainsi dire autonome, détachée du cursus de traduction proprement dit. Or le développement de la «théorie interprétative de la traduction»(TIT) a bien montré qu'il y a un 
noyau commun important à la base du travail du traducteur et de l'interprète (Seleskovitch et Lederer, 1993). Ce qui apporte une confirmation au fait - longtemps sous-estimé par les spécialistes et toujours méconnu du public - que la traduction (ainsi que l'interprétation) n'est pas exclusivement une affaire de langue, mais de saisie du sens.

Bien plus, nos cursus sont maintenant confrontés au défi de la formation à la communication multimédia. Cela implique d'abord la maîtrise d'un « outil » technologique spécifique, mettant en œuvre plusieurs niveaux et plusieurs supports du message à traduire. Cela entraîne du même coup une diversification des types d'équivalences traductives attendues, sur lesquelles il faudra jouer en fonction de l'économie globale du produit final. Il faut en outre intégrer la contrainte aggravée du facteur temps; et Yves Gambier va même jusqu'à prendre en compte les incidences que cela comporte dans le travail de médiation interlinguistique, quitte à ce qu'il faille faire son deuil du perfectionnisme traditionnel des traducteurs (lui-même corollaire du privilège de l'écrit, qui vient d'être évoqué [Gambier, 2000]).

En somme, il s'agit moins d'ignorance que de ce que l'on pourrait appeler des «connaissances-écran» (au sens où Freud parle de «souvenirs-écran») qui enferment le client dans une méconnaissance de la compétence réelle du traducteur du fait d'une conception historique et restrictive de la traduction.

Ce qui est en jeu dans l'acte de traduire exige et permet d'aller bien au-delà du texte ou de l'écrit: le traducteur est celui qui transmet du sens, et qui intervient comme médiateur entre les langues et les cultures. À ce titre, les compétences acquises dans une formation à la traduction dépassent largement la stricte compétence linguistique (langues $\mathrm{ABC}$ ou $\mathrm{ABB}$, voire $\mathrm{AA}$ ') et même la sémiotique différentielle des textes (To et $\mathrm{Tt}$ ).

Résoudre l'ensemble de ces questions liées au transfert de sens d'une langue ou d'une culture à l'autre, c'est plus fondamentalement gérer du transfert interculturel. À vrai dire, cette idée est très souvent évoquée mais rarement développée : il conviendra d'en dégager les implications concrètes sur le plan de la formation des traducteurs et des interprètes (Ladmiral et Lipiansky, 1991).

La polysémie de ce «concept-valise» qu'est la traduction (lato sensu) a paradoxalement pour effet d'interdire l'accès à ce qu'il désigne. On conçoit aussi qu'il soit excessivement difficile, sinon impossible, de proposer une définition de la traduction. À la limite, la traduction est un concept qui tend à faire figure d' «indéfinissable» du système (au sens de l'axiomatique) (Ladmiral, 2002). La traduction est une pratique complexe et différenciée qui, en dernier recours, ne s'autorise que de l'expérience. Pour tout dire: un traducteur est à même de tout traduire (textes, images, logiciels, boutons...) parce que justement il traduit! Pour un Daniel Gouadec (2002), « [...] la liste est infinie: tout ce qui se communique - pas seulement par des moyens linguistiques - est susceptible de traduction ».

Il y a sans doute plusieurs réponses à apporter aux différents problèmes ainsi posés.

La première attitude consiste à dire: «Il suffit d'insister!» pour reprendre une formule dont usent cum grano salis (et a contrario) les psychologues comportementalistes face aux conduites d'échec compulsives. Il s'agirait dès lors de s'acharner à essayer de convaincre les récalcitrants que l'appel à un traducteur professionnel est incontournable, quelles que soient les objections formulées (et les coûts occasionnés), 
et que toute autre solution est une impasse qui, finalement, reviendra encore plus cher. Il n'y a que la foi qui sauve!

La deuxième attitude consiste à substituer à l'étiquette de «traducteur» la qualification d'expert en communication multilingue et multi-support, d'expert en communication internationale; on pourra parler aussi de traduction stratégique pour les entreprises, etc. En résumé, il s’agit de développer des concepts pour leur donner une dimension descriptive qui viendrait suppléer à la trop grande généralité du vocable «traducteur». Mais n'y doit-on pas voir une «valse des étiquettes» qui rendrait plus inintelligible encore la nébuleuse des professions concernées?

Une troisième attitude irait à «coller au marché». Il s'agira dès lors de développer des compétences qui correspondent aux attentes du marché telles qu'elles s'expriment ou sont censées s'exprimer. Avec un double risque: celui d'avoir des attentes d'autant plus mal définies que les besoins réels ne sont pas connus du marché lui-même; ou celui de mettre en œuvre, au bout du compte, des compétences strictement techniques, lesquelles ne sont plus guère des compétences en traduction. Mais n'est-on pas amené à évoquer mutatis mutandis la formule de Valéry: "il n’y a que les huîtres et les sots qui adhèrent ».

Toutes ces attitudes ont un sens mais elles posent toutes problème. En tout cas, il faudra envisager d'inclure ces questions dans la conception même de la formation de traducteurs.

En somme: la relation qu'entretiennent le traducteur et son marché est une relation assez largement brouillée. Il s'agit donc de contribuer à la clarifier; et, dès lors la question se pose de savoir quel peut en être l'impact sur la formation. Il nous est apparu que toutes les distinctions conceptuelles que l'on veut établir entre les concepts de traduction, d'adaptation, de localisation, de transfert linguistique... apportent certaines clarifications mais engendrent parallèlement d'autres confusions. Le terme générique reste la traduction; et les distinctions conceptuelles devraient apparaître à propos des «outils» de travail et des «objets» à traduire: logiciels, images, textes, contrats, etc. En effet, il est certes impératif que les cursus de formation à la traduction professionnelle prennent en compte l'ensemble des données (et des contraintes) qui viennent d'être évoquées, sans pour autant provoquer les ambiguïtés que nous venons de soulever. Il s'agit toujours de formation à la traduction, ce qui implique notamment une gestion du décalage interlinguistique et interculturel, sans parler des exigences de la maîtrise de la langue maternelle.

Cela dit, une formation adéquate aux transformations du marché doit intégrer de nouvelles compétences en matière d'informatique, de multimédia, et autre, NTIC (nouvelles technologies de l'information et de la communication). Mais ce ne sont pas des formations spécifiques venant suppléer la formation à la traduction et créer par là même ce qui constituerait de nouveaux métiers, comme la «localisation», «l'adaptation», la rédaction technique, etc. Ainsi formés, nos étudiants sauront implicitement qu'ils ont acquis des outils et non pas de nouveaux métiers. Faute de quoi, une adaptation au marché trop immédiate conduirait à former des compétences partielles qui ne seraient pas des compétences en traduction ou, inversement, la non-prise en compte de ces nouveaux «outils» rendrait les étudiants incapables de s'insérer dans la vie professionnelle.

Du point de vue du client, l'accent mis sur ces formations complémentaires lui permettrait de mieux prendre en compte la spécificité et les enjeux de la traduction 
elle-même et ainsi d'éviter les écueils et les confusions dont il a été question plus haut.

Rajouter un «étage» de formation technique à l'édifice d'un cursus de traduction déjà complexe voilà qui n'est pas sans avoir des répercussions sur l'ensemble, notamment sur le niveau de qualification attendu et donc sur la durée de la formation. Au reste, la Convention de Bologne va au-devant de ces considérations en proposant une distinction entre bacc. +3 (licence) et bacc. +5 (master) au sein des formations professionnelles, comme celle qui nous concerne. Si d'aucuns tenaient à mettre en place des licences professionnelles dans l'univers de la traduction, quel pourrait bien être le niveau de qualification auquel ils pourraient raisonnablement prétendre?

Pour nous faire mieux comprendre, nous userons d'une analogie. En français, on distingue l'ingénieur du technicien, non seulement par la durée respective de leurs études (bacc. +5 et bacc. +3 ), mais aussi du même coup par la complexité des compétences acquises: un ingénieur conçoit, un technicien applique. Une licence professionnelle conduira à des métiers techniques d'application: «localisateur», «communicateur bilingue», etc. Le master professionnel en traduction conférera des compétences suffisamment larges pour recouvrir l'ensemble des activités mises en jeu dans la traduction (comme un ingénieur les a en physique, informatique, chimie...). La maîtrise des nouveaux outils techniques s'y inscrira comme un prolongement direct, qui fait partie intégrante du métier. Si nous croyons devoir écarter l'éventualité d'une formation courte et strictement technique, c'est parce que le bagage langagier et culturel d'un traducteur implique une formation longue et une certaine maturité de l'apprenant, d'une part, et parce que l'évolution inévitable des métiers de la traduction et de leurs composantes techniques (et ce, dans un très proche avenir) exige davantage une aptitude à concevoir, à comprendre et à anticiper qu'une capacité à appliquer et à réaliser, d'autre part.

C'est pourquoi il y a sans doute lieu d'être tout à fait réservé à l'égard de projets, qui s'annoncent ici et là, de mettre en place des licences professionnelles dites «bilingues", voire «trilingues", dont les débouchés annoncés pourraient laisser croire qu'ils concernent aussi la traduction, quels que soient les étiquetages sous lesquels on entend les «vendre» aux demandeurs de formation. Il n'en va pas autrement dans les cas où, inversement, on prétendrait attribuer un "master de traduction » au terme de formations diverses incluant des enseignements de langues étrangères, que viendrait seulement «couronner» un cycle d'un an censé conférer le faisceau des compétences attendues d'un véritable traducteur. Inversement, il ne suffira pas d'un simple supplément linguistique pour conférer un statut de «traducteur» au détenteur d'un master d'une discipline quelconque, technique ou non. Sur le plan didactique, rappelons que les langues sont la condition nécessaire bien sûr, mais pas la condition suffisante, de toute traduction; et surtout que les exercices dits de traduction pratiqués dans le cadre de l'enseignement des langues n'ont qu'une fonction pédagogique d'apprentissage et n'ont pas grand-chose à voir avec la pratique de la «traduction traductionnelle» (Ladmiral, 2002). Et là encore, inversement, on peut dire la même chose concernant des licences professionnelles centrées sur des outils techniques, comme l'informatique, auxquelles on se sera contenté de rajouter quelques enseignements de langues (et pour mieux dire d'anglais!) en s'imaginant qu'ils pourront «localiser», «adapter», etc. 
Le fait que la traduction soit un concept-valise, comme nous l'avons défini d'entrée de jeu, a des incidences très concrètes. Ce flou conceptuel est à l'origine de brouillages sur le plan du marché, comme il a été indiqué. Mais il y a plus grave: certaines formations qui, pour des raisons d'opportunisme et de recherche de débouchés, s'inventent des compétences en traduction qu'elles n'ont pas, contribuent à une telle confusion et semblent vouloir en exciper pour s'exonérer des exigences attachées à une formation digne de ce nom. C'est pourquoi il nous est apparu qu'au-delà d'une simple question de mot, il fallait dévaliser le concept !

\section{RÉFÉRENCES}

GAmbier, Y. (2000) : «Le traducteur des multi-médias: une nouvelle identité» in MEJRI, S., A. Clas, T. BAccouche et G. Gross (éds.) : La traduction: théories et pratiques, Tunis, École normale supérieure, vol. I, p. 111-121.

Gouadec, D. (2002): Profession: traducteur, Paris, La Maison du Dictionnaire.

Ladmiral, J.-R. (1995) : «Traduire, c'est-à-dire...? Phénoménologies d’un concept pluriel», in Meta 40-3, p. 409-420.

LAdmiral, J.-R. (2002): Traduire: théorèmes pour la traduction, Paris, Gallimard, coll. «Tel », nº 246. Le traducteur et l'ordinateur: Numéro thématique de la revue Langages, ${ }^{\circ}{ }^{116}$, décembre 1994.

Ladmiral, J.-R. et E. M. Lipiansky (1991): La Communication interculturelle, Paris, Armand Colin.

Mounin, G. (1955) : Les Belles Infidèles, Presses Universitaires de Lille, rééd. 1994, coll. «Étude sur la Traduction».

Seleskovitch, D. et M. Lederer (1993): Interpréter pour traduire, Paris, Didier Erudition, coll. «Traductologie». 